\title{
The Lived Experiences of Mothers of Children with the Autism Spectrum Disorders in Egypt
}

\author{
Ereny Gobrial ${ }^{(1)}$ \\ Mental Health Department, Faculty of Education, Zagazig University, Shaibet an Nakareyah, \\ Markaz El-Zakazik, Ash Sharqia Governorate 44519, Egypt; ereny.gobrial@hotmail.co.uk
}

Received: 23 June 2018; Accepted: 9 August 2018; Published: 12 August 2018

\begin{abstract}
The aim of this study was to gain insight into the experiences of mothers caring for children with autism spectrum disorders (ASD) in relation to the early life, resources and to address the consequences of raising a child with ASD in Egypt. Semi-structured interviews were conducted with 14 mothers of children with ASD in Egypt, the children were aged 5-14 years old (mean: 7.3 years). Data were thematically analysed. Results revealed that life with ASD was daunting for the Egyptian mothers. Findings suggested that provision of inadequate education, healthcare and stigma constitute the main issues for mothers. Furthermore, ASD impacted negatively on the social life, emotional wellbeing and sacrifices of mothers of children with ASD. The findings provided valuable insight into the life of mothers, revealing what life really is like for mothers caring for a child with ASD in one of the low-medium-income countries. Understanding the mothers' experiences of caring for children with ASD is crucial in providing support and developing the services that are urgently needed in Egypt.
\end{abstract}

Keywords: autism spectrum disorder; children; Egypt; family; mother

\section{Introduction}

Mothers experiences raising a child with autism spectrum disorder (ASD) conveys a complex and extremely challenging life. ASD is a lifelong developmental disability characterised by qualitative impairments in three domains: social interaction, communication and repetitive, stereotyped behaviour (APA 2013). There is considerable evidence that shows the incidences of ASD has increased throughout the world as diagnostic and screening techniques become better developed. Approximately 52 million individuals have been reported to be on the spectrum globally (Baxter et al. 2015). As the prevalence of individuals with ASD has increased, a new population of families has emerged which presents further challenges as our knowledge increases. Despite that the characteristics of ASD being commonly found across cultures, the family experiences of caring for a child with ASD could be varied (Riany et al. 2016; Kim 2012; Divan et al. 2012). It is important to study the experience of mothers of children with ASD, nationally and internationally, in order to inform the policy and legislative process (Preece and Jordan 2010).

The core ASD features, associated symptoms and behaviour problems of the ASD perhaps will cause significant negative impact on families and parental wellbeing. A significant body of research has illustrated the immense challenges to the individuals with ASD and their families and the impact on the parental well-being this included: emotional stress, ongoing financial burden of expensive treatments and therapies, significant strain on family relationships, changes in family roles, structure and activities, feeling of guilt and blame regarding diagnosis and social stigma (DePape and Lindsay 2014; Weiss et al. 2014; Smith et al. 2012; Ekas and Whitman 2010; Altiere and Von Kluge 2009; Schieve et al. 2007). Previous research has stated the profound impact of caring for a child with ASD on mothers (Opreaa and Stan 2012; Koydemira and Tosuna 2009). Much of the available research on these issues, however, stems from western societies, particularly the UK and USA. 
Research studies concerned the parental experiences during diagnosis, in the UK, indicated an average delay of 3.5 years for ASD diagnosis, referring that half of the parents surveyed were dissatisfied with the diagnostic process (Crane et al. 2016; Keenan et al. 2010). While Reed et al. (2016) reported negative impact of diagnostic practices on mothers related to levels of anxiety and depression. They suggested that providing information and help sources throughout the process, may improve the mother's perception of the process and reduce the negative impacts of the diagnosis on the family. Dickinson and Place (2016) concerned a parental stress, illustrated that mothers of children and adolescent of ASD in the UK experienced high levels of stress, in favour of the mothers of younger children, with the parenting of teenage girls being the most stressful. Mothers in this study reported a low level of satisfaction with family life.

Limited research has examined the experience of caring for children with ASD in Low-mediumincome countries (LMICs) and non-Western countries compared to high-income countries (For example, Ilias et al. 2017; Blake et al. 2017; Tilahun et al. 2016; McKenzie and McConkey 2015). A recent qualitative study by Ilias et al. (2017) investigated the experiences of mothers' wellbeing of children with ASD in Malaysia. The authors illustrated that Malaysian mothers viewed their child's ASD symptoms and behaviour problems as taking a toll on wellbeing. They reported mothers' coping strategies, included acceptance, proactive mindset, character growth, spirituality, parent support networks and fostered wellbeing.

In Egypt, caring for a child with ASD can be daunting and overwhelming for mothers, due to the services for children with disabilities in general, and services specifically designed for children with ASD in Egypt, are minimal or even absent. Children with ASD and their families face the likelihood of poor health, social care, mental health service, rehabilitation, lack of special education and access to equal opportunities (Gobrial et al. 2018; Omar 2014; Gobrial 2012; Jenkins et al. 2010; Okasha 2005). The majority of individuals with ASD in Egypt remain at home uneducated. They are given little to no education because they either drop out of mainstream schools or parents cannot afford the scarce and expensive private schools. Meanwhile, parents receive a low level of support to their children with ASD (Omar 2014; Mendoza 2010). Thus, Egyptian mothers of children on the autism spectrum are expected to experience greater burdens. Omar (2014) investigated the early intervention services as perceived by parents of children with ASD in Egypt and Saudi Arabia, reported a low level of parent's perception for early intervention and a lack of early intervention services provided to children with ASD.

It is crucial to unravel the mothers' experiences of caring for a child with ASD to better facilitate adequate and effective interventions. Research focused on the experiences of mothers caring for people with ASD in Egypt is largely unexplored and rarely addressed. The purpose of this study was to gain insight into the experiences of mothers caring for children with ASD in Egypt in relation to the early life of ASD, resources and to address the consequences of raising a child with ASD in Egypt.

\section{Method}

The study adopted a qualitative approach, a grounded theory was applied, which provided insights into the lived experiences of mothers of children with ASD. Data were analysed using the thematic content analysis (Braun and Clarke 2006) through the constant comparative method, applying coding with MAXqda software.

\subsection{Participants}

The participants were comprised of fourteen mothers, all of whom were caring for a child with ASD aged between five and 14 years (mean: 7.3 years, SD: 3.2 years), mainly boys (12 out of 14). Seventy-eight per cent of the children received a diagnosis of autism, the remaining had no confirmed diagnoses. Almost two-thirds of children (9 out of 14) were out of school, and the other children (5 out of 14) were attending either a private school or a private day care centre and all children reside at home (details shown in Table 1). All fourteen families who expressed interest in taking part in this study were involved. 
Table 1. Socio-demographic characteristics of study participants.

\begin{tabular}{|c|c|c|c|c|c|c|c|c|}
\hline Child & Child Gender & Child Age & ASD Diagnosis & Other Disabilities & $\begin{array}{l}\text { Employment } \\
\text { Status }\end{array}$ & Birth Order & School Type & Material Status \\
\hline 1 & Male & $10 \mathrm{Y}, 6 \mathrm{M}$ & Autism & No & Housewife & Only son & No School & Married \\
\hline 2 & Male & $5 \mathrm{Y}, 6 \mathrm{M}$ & No confirmed diagnosis & No & Housewife & Last of 5 children & Private day care* & Widow \\
\hline 3 & Female & $6 \mathrm{Y}, 3 \mathrm{M}$ & Autism & No & Housewife & Last of 3 girls & Private day care & Married \\
\hline 4 & Male & $6 \mathrm{Y}, 5 \mathrm{M}$ & Autism, & No & Employment & Last of 6 children & No School & Married \\
\hline 5 & Male & $8 \mathrm{Y}$ & Autism & No & Housewife & 2nd of 2 boys & No School & Married \\
\hline 6 & Male & $7 \mathrm{Y}$. & No confirmed diagnosis & No & Employment & 1 st of 2 boys & No School & Married \\
\hline 7 & Male & $5 \mathrm{Y}$. & Autism & No & Employment & Middle son of 3 boys & Private school & Married \\
\hline 8 & Male & $12.5 \mathrm{Y}$ & Autism & No & Employment & 1st of 2 boys & No School & Married \\
\hline 9 & Female & $14 \mathrm{Y}$ & Asperger's syndrome & No & House wife & 2nd of 2 girls & Private school & Married \\
\hline 10 & Male & $5 \mathrm{Y}, 3 \mathrm{M}$ & Autism & No & Housewife & Only son & No School & Married \\
\hline 11 & Male & $8 \mathrm{Y}$. & No confirmed diagnosis & No & Employment & Only son & No School & Married \\
\hline 12 & Male & $6 \mathrm{Y}$. & Autism & No & Housewife & Last of 4 children & * Private day care & Widow \\
\hline 13 & Male & $6 \mathrm{Y}, 6 \mathrm{M}$ & Autism & No & Housewife & Only son & No School & Married \\
\hline 14 & Male & $5 Y, 6 \mathrm{M}$ & Autism & Hydrocephalus. & Housewife & 2 nd of 2 children & No School & Married \\
\hline Total & $\begin{array}{l}\text { Male }=12 \\
\text { Female }=2\end{array}$ & $\begin{array}{l}\text { Mean }=7.3 \\
\text { S. D }=3.2\end{array}$ & $\begin{array}{c}\text { Autism confirmed diagnosis }=10 \\
\text { Aspergers }=1 \\
\text { No confirmed diagnosis }=3\end{array}$ & $\begin{aligned} \text { No other disability } & =13 \\
\text { Other disabilities } & =1\end{aligned}$ & $\begin{array}{c}\text { Housewife }=9 \\
\text { Employment }=5\end{array}$ & $\begin{array}{l}\text { Only child }=4 \\
\text { First child }=2 \\
\text { Middle child }=1 \\
\text { Last child }=7\end{array}$ & $\begin{array}{c}\text { Private day care }=5 \\
\text { Out of school }=9\end{array}$ & $\begin{array}{l}\text { Married }=12 \\
\text { Widowed }=2\end{array}$ \\
\hline
\end{tabular}

* Private day care is a special needs day nursery for children with disabilities. 


\subsection{Measures}

To obtain qualitative data about the life of mothers of children with ASD, a semi-structured interview schedule was developed as a guiding framework with open ended questions (see Appendix A). The interview questions sought to explore the mothers' experiences to get rich details of the experiences of living with ASD children. The main questions of the interview delve into mothers' view of ASD since the early days before their children received any diagnosis, through the milestone of the diagnosis to the moment. The interviews took between 60-90 min and were in Arabic, the native language of the Egyptian.

\subsection{Procedure}

Ethical approval for the study was granted from the local University Research Ethics Committee. Information was provided and written informed consent obtained from all participants who were recruited from two local charities, a private day care centre for children with disabilities \& ASD and a speech therapy centre, both located in Zagazig city, Sharkyia Governorate in the north-eastern region of Egypt.

The interviews were recorded on a digital recorder. Subsequently, the recordings of the interviews were transcribed, anonymised and entered into the computer software package MAXqda, which supports a thematic approach to analysis.

\section{Results and Analysis}

The present study investigated the lived experiences of the Egyptian mothers caring for a child with ASD in Egypt. Eight thematic concepts were identified, which included: diagnosis, resources (health resources and education), financial strain, emotional well-being, personal sacrifices, impact on social life, social \& emotional support and cultural influences.

\subsection{Diagnosis}

The findings revealed the mothers' experiences about diagnosis, misdiagnosis and a lack of awareness among both mothers and professionals. 11 out of 14 of the interviewed mothers noticed difficulties in their children by 12 months of age. The average age at which concerns were first noted was at 18 months. The mothers' concerns involved delay or a motor impairment, difficulty with speech and language, non-verbal communication difficulties, a ritualistic and repetitive repertoire of behaviour (flapping hands, spinning, echolalia), attachment and attention deficits. Other mothers indicated a regression including communication and social skills, between 12 and 24 months. However, they often struggled to get a diagnosis of ASD and spent years pursuing an explanation. Mother (9) reported:

"When she was 18-month-old, she began isolating herself, sitting down in the corner, lining up her toys in a pattern in a semi-circle, this can last for hours. Gradually, she stopped talking, then she became isolated completely." (Mother 9).

8 out of 14 mothers also reported that their children had received a misdiagnosis at some point. Although mothers were significantly more likely to report early recognition of the difficulties, professionals were unable to recognise it as ASD. This frequently led to misinterpretation, with signs associated with ASD considered evidence of 'behaviour problem', 'Anaemia', 'hearing impairment', 'lack of calcium', 'attention deficits' or 'hyperactivity'. The subsequent quote reveals both misdiagnosis and the lack of awareness. Mothers stated:

"Initially, we had been told that he has anaemia, which had impacted on him and caused speech problems." (Mother 13).

"He had an incorrect diagnosis of hearing impairment. Hence, he received incorrect treatment for five years." (Mother 11). 


\subsection{Resources}

The major concern to the Egyptian mothers was resources and services provided to children with ASD in Egypt. All interviewed mothers emphasised the absence of important services for children with ASD, for example, health services, rehabilitation and social care services. Mothers reported that there were extremely limited, or no services provided, or that they were only available in private clinics in the capital city.

Mothers indicated the need for appropriate educational provision specifically aimed at children with ASD as well as access to a range of support and services to meet their children's needs. Furthermore, the findings revealed that the $64 \%$ (5 out of 14 ) of children were at home with no education. Children were excluded from schools, mothers reported:

"Despite that my son reaching the age he should start school, no school offered him a place, neither at the intellectual disabilities nor the hearing and speech impairment schools. Unfortunately, when I just mention that he has some difficulties, the school refused to offer him a place! Where shall I go?" (Mother 7).

"My son was rejected from every school I applied for because he needs a special care. I lost hope, no way will he be enrolling in a school. He is at home, uneducated." (Mother 5).

All Egyptian mothers indicated the need for appropriate educational provision for their children. Consequently, others cited developmental learning obstacles and ostracism in keeping children with ASD at home.

\subsection{Financial Strain}

Finance is considered a major concern for mothers caring for a child with disabilities in Egypt. The majority of the mothers ( 8 out of 14 ) reported financial difficulties due to the intense and costly interventions, speech therapies and private special education. Moreover, 5 out of the 14 interviewed mothers reported that they could not afford the highly expensive medication for a long-term condition. Some mothers stated that they had to end their child's speech therapy and medication due to financial difficulty. The mother (14) reported:

"The medication and speech therapy and travelling to Cairo [for services] are too expensive. It was overwhelming. It exceeded his father's financial resources. His father left his career and worked as a driver to earn money, which is still not enough." (Mother 14).

\subsection{Emotional Well-Being}

The findings suggested that all mothers experienced poor emotional well-being and feel depressed about their children's conditions. This included: feelings of being overwhelmed; despair at the incurable nature of the disorder; guilt that something they did may have caused their child's challenges and frustration that the parenting experience they had is not what they envisioned. 6 out of the 14 participated mothers reported the mental health consequences, arising because of the massive change in their life due to ASD. For example, mother reported:

"There is no way out! I am so distressed and depressed." (Mother 1).

\subsection{Personal Sacrifices}

Mothers cited the influence of ASD on their professional career. Some mothers (5 out of 14) indicated that they had to leave their jobs to care for their children since the day-care centres were either expensive or not available. They indicated that they made major sacrifices to look after their ASD child and resigned. On the other side, their fathers had to work extra hours or two jobs to cover the expenses caused by ASD. Mothers stated: 
"I took 4 years of work to care for my son at home." (Mother 6).

"I am a physician, however, I had to resign. I did not leave my house for over 10 years since my son was born. This is because I am really busy looking after him, going to doctors, speech therapy and travelling to Cairo." (Mother 1).

Furthermore, mothers indicated that children with ASD have a disturbed their sleep pattern and they needed constant supervision, which was physically exhausting for them. For example, mothers explained;

"I'm awake all the time, I cannot sleep because he is awake all the time. If he falls asleep for 15 min, he will be awake for 3 days continuously!" (Mother 7).

\subsection{Impact on Social Life}

Mothers revealed feeling socially isolated and embarrassed about their child's behaviour in public. Therefore, half the mothers ( 7 out of 14) made proactive choices, preferring to keep the child at home by making schedules for others to care for the child at home in case of a social event (e.g., birthday, wedding), based on anticipating recurrences of previous negative experiences. They reported a negative impact on the entire families' and children's social life. For example, one mother explained:

"Of course, there is a profound impact. For example, if we are going to attend a wedding or a party, we usually think hundreds of times before we decide to go and plan for when he [the son] gets bored who will help! I mean dad, grandma and I; we call us the triangle." (Mother 6).

Other mothers reported avoiding taking their children to public places rather than risk public judgment when their child becomes anxious. This not only caused the child with ASD but also any siblings to become housebound and isolated, which has a profound effect on their social and emotional well-being. Mothers who are housewives were more likely to feel socially isolated than the employed mothers.

\subsection{Social \& Emotional Support}

The interviewed mothers indicated that they were trying to cope with a condition that they did not know, or may know very little, about. They tried to find a way to support their children with ASD. Mothers were trying to educate themselves and empowered themselves to help their children and implementing coping strategies at home. They also reported being given positive support-10 mothers out of the 14 stated being given emotional support from their husbands. One mother reported:

"My husband was a great support to me. Maybe he is stronger than me! Yes, he is. I admit, when I was so frustrated he was always there next to me and got my back." (Mother 6).

Additionally, the extended family showed empathy and expressed positive social support towards their family member with ASD and provided the help and support to care for the child.

\subsection{Culture Influences}

\subsubsection{Culture Believes}

There is no doubt that culture influences parental belief systems and explanatory models for disorders such as ASD. In the current study, Egyptian mothers had a favourable view of ASD, believing it is a blessing or a gift from God. Mothers showed strong religious belief in God and hope in their children. In the following extract, mothers described their beliefs:

$$
\text { "God granted me this girl ..." (Mother 3); }
$$


Another faithful mother said: "He is my only son, along with the other five daughters. Whatever he is, he is a gift from God. I call God to strengthen me and I ask for patience. I am satisfied" (Mother 4).

"He is a gift from God" (Mother 10).

10 out of 14 mothers expressed their fears and worries about their children's future, when they are older and no longer able to support them. Mother reported:

"What worried me was that when I get older and die, who would care for him?" (Mother 5).

\subsubsection{Social Stigma}

Egyptian mothers experienced stigma, 8 out of 14 mothers reported that their ASD children were treated differently by society. Others felt embarrassed about their child's behaviour or ashamed of the condition. Half of mothers (7 out of 14$)$ reported that they prevent their child to go to play with their relatives, worried that their child would behave inappropriately. Because ASD is not a visible physical disability, most people condemn 'odd' behaviour and attribute everything to bad parenting or the mothers not controlling their child. Consequently, mothers reported that they were forced to hide with their autistic child at home and feeling socially isolated. A mother explained:

"When we go out, people in the street stare at us in a weird way, as we are coming from a different planet! Furthermore, a mother may take away her child from my child because the child may like to play with my son." (Mother 1).

\section{Discussion}

This study sought to explore the lived experiences of mothers caring for a child with ASD in Egypt. The findings demonstrated the negative aspects of mothers' experiences of having a child with ASD. It varies from the poor knowledge to the lack of specific resources, ongoing financial burden, feelings of social isolation, stigma and personal sacrifices. The consequences of ASD were quite profound on the life of mothers of children with ASD in Egypt. However, regardless of how difficult their life with ASD, mothers tried to cope with the ASD and they clearly reported that they are committed to creating opportunities for their children to thrive. The findings provide valuable insight into the life of mothers but also, reveal what life is really like for mothers caring for a child with ASD in Egypt.

The lack of awareness of ASD reported in this study was previously described in the literature concerned LMICs (Ilias et al. 2017; Neik et al. 2014). Delay in diagnosis has also been reported in this study between initial concerns and the child receiving a diagnosis of ASD-this is concordant with the previous research (Crane et al. 2016; Keenan et al. 2010).

The findings suggest that the inadequate provision of adequate education, health care and other resources constitute the main issue in relation to ASD in Egypt. The difficulty in accessing ASD specific interventions proved challenging for children with ASD in Egypt, as well as for their mothers. Insufficient attention has been given to special education services and health services for children with ASD in Egypt, resulting in no holistic view of the child's and families' need. This is in line with prior research referring to the lack of access to health-care and special education services generally in the Arab countries and specifically in Egypt (Gobrial et al. 2018; Hadidi and Khateeb 2015; Omar 2014; Gobrial 2012; Jenkins et al. 2010). Also, this is consistent with the previous literature concerned limited resources, underdeveloped health systems and scarce knowledge of ASD those living in lowto middle-income countries (Blake et al. 2017; Tilahun et al. 2016; McKenzie and McConkey 2015; Iemmi et al. 2016).

Special education is considered a crucial aspect in ASD treatment. In line with the global trend of inclusion education (IE), Egypt is preparing the school to accept children with ASD in the mainstream school. The concept of this IE deems that children with mild disabilities (list includes ASD) shall have the right to enroll in any mainstream education (Al-Masry Al-Youm 2017). Despite that, mothers 
participated in this study indicated that their children were rejected from all type of school, mainstream and special school. Findings indicated that the most of school age children with ASD in Egypt were left at home, uneducated. Furthermore, less support was given, mostly by the government, regarding mainstreaming children with ASD within the educational system. This is also consistent with the available data reported on the need for special education that meets the children's needs in Egypt (Gobrial et al. 2018; JICAPED 2002). The introduction of this service could dramatically influence the life chances of Egyptian children with ASD.

The current study demonstrated that all mothers of children with ASD caring for their dependent children at home had no form of financial nor professional support. Financial strain appeared as a barrier to appropriate care for children with ASD in Egypt. In Egypt, due to the absence of national health services for children with ASD, and disabilities in general, it is more common for parents to seek treatment from privately owned clinics - though parents are expected to pay for those services themselves. Hence, even the wealthiest families could provide the circumstances for their children to flourish via private education or intervention.

The findings referred to the mother's well-being and parental stress as a result of the ASD. This is in line with the literature reporting that bringing up a child with ASD often associated with a plethora of difficulties for caregivers, including feelings of being overwhelmed, increased parenting stress and mental health problems compared with mothers of both typically developing children and children with other developmental disorders (Omar et al. 2017; Weiss et al. 2014; Fido and Al Saad 2013; Opreaa and Stan 2012; Karst and Van Hecke 2012; Koydemira and Tosuna 2009).

The results identified the social and emotional support given such as the father always being the main support to the mother, both parents becoming united and closer as a family. This is also reported in the literature (Bayat and Schuntermann 2013; Hock et al. 2012). Alongside this, the extended Egyptian family (mainly grandparents) played a vital role in the family dynamics providing an incredible social support from family member to their grandchildren, which was important in facing the challenges of caring for a child with ASD, particularly where special education is not available or unaffordable-Egyptian mothers appeared more likely to rely mainly on the grandparents' support. This may be in response to the lack of adequate support for individuals with disabilities. In Egypt, individuals with disabilities depend solely on their families for support and must eventually face a time when their parents will be no longer there for them.

Findings identified that mothers were religious and gaining spiritual strength, which helped them to accept their child's disabilities — this is in agreement with the literature aimed to explore a religious perspective among Asian Muslim families indicating that mothers regarded their children as gifts from God and felt blessed that God chose them to be special parents (Jegatheesan et al. 2010). Mothers also expressed their views on the effect of social stigma on their life with ASD children. As a result, mothers with ASD felt socially isolated as they didn't engage in social activities and kept their ASD children at home which is consistent with previous research (DePape and Lindsay 2014; Divan et al. 2012; Altiere and Von Kluge 2009). In turn, this could have negative impact on their family welling (Smith et al. 2012; Patterson and Smith 2011; Ekas and Whitman 2010). Despite that the current findings indicated social stigma among mothers of children with ASD, previous research showed positive societal attitudes towards individuals with ASD in Egypt (Gobrial 2015).

\section{Limitations, Implications and Future Directions}

It is important to address the limitations of this research. The mothers who participated in this study were recruited from one geographic area, recruitment from a broader geographic region (including remote and rural areas) would help provide a range of experiences of mothers in Egypt. This study represents mothers' experiences from a limited age group (children), additional research extends to a range of ages, for example, adolescents and adults with ASD, would be helpful to provide more experiences from other age groups. Insights from participants recommend that providing information and appropriate sources to mothers may help reduce the profound impacts on the mothers 
and improve the family wellbeing. Findings highlighted the importance of developing family-centred services that enhance parenting skills and support mothers in coping with distressing emotions and obstacles. Nevertheless, this paper reflects the lived experience of mothers of children with ASD in Egypt, which can serve as a guide in improving and delivering services to children with ASD.

The findings of the current study suggest additional studies would be beneficial to support mothers with children with ASD and would enhance their wellbeing. Overall, there was a consensus that future priorities for ASD research should lie in areas that make a difference to people's day-to-day lives.

\section{Conclusions}

This study provided valuable insights into the mothers' experiences of caring for children with ASD in Egypt. The findings of this study reported a range of adverse impacts associated with caring for a child with ASD in Egypt, this included: financial strain, emotional wellbeing, personal sacrifices of mothers and socially isolation. Findings suggested that lack of awareness, inadequate provision of education and healthcare as well as social stigma constitute the main issues for mothers of children with ASD in Egypt. This study contributes to the entire global ASD literature by exploring the experiences of mothers of children with ASD in Egypt.

Revealing the mothers' experiences would lead to better services and encourage the development of a set of standards for the assessment and diagnosis of ASD in children across Egypt. It is necessary that the mothers and their children with ASD should have the same opportunities as everyone else for a fulfilling and prosperous life. This study urges the Egyptian government to provide the support that children with ASD and their families so desperately need and call for the development of free to access services to children and families in Egypt, such as early diagnosis, counselling parents, education of school aged children, community living arrangements for adults, social and leisure time activities.

Funding: This research received no external funding.

Acknowledgments: The author would like to thank all the families who participated in this study and shared their personal experiences.

Conflicts of Interest: The author declares no conflict of interest.

\section{Appendix A. Semi-Structured Interview Guide and Questions}

(1) Could you please tell me about the early life of your child with ASD?

(2) Tell me about the milestones of diagnosis development: e.g., when was the first concern of abnormalities with your child ... ?; how old was s/he? Where did you go ... ? How it went? How does it affect you the member of the family being diagnosed with ASD?

(3) How did the diagnosis of ASD impressed you? Your husband? Your family (mum \& dad)?

(4) Tell me the experience of the SEN education services?

(5) Intervention: was there any intervention? Was there any change? How did it change?

(6) Tell me about you as a mother of ASD! Is there any point in those years where you felt like giving up? Were you frustrated, were you angry?

(7) Tell me about your experiences as a mother of a child with ASD in the Egyptian culture and the relationship between ASD and society.

(8) Can I finally ask you if you think there is any other aspect of your experience of ASD you would like to add! 


\section{References}

Al-Masry Al-Youm. 2017. 800000-Egyptians-Estimated-Have-Autism-Social-Solidarity-Minister. Available online: http:/ / www.egyptindependent.com/800000-egyptians-estimated-have-autism-social-solidarityminister/ (accessed on 30 September 2017).

Altiere, Matthew J., and Silvia Von Kluge. 2009. Family functioning and coping behaviours in parents of children with autism. Journal of Child and Family Studies 18: 83-92. [CrossRef]

American Psychiatric Association (APA). 2013. Diagnostic and Statistical Manual of Mental Disorders-5 (DSM-5). Washington: American Psychiatric Association.

Baxter, A., T. Brugha, H. E. Erskine, R. W. Scheurer, T. Vos, and J. G. Scott. 2015. The epidemiology and global burden of autism spectrum disorders. Psychological Medicine 45: 601-13. [CrossRef] [PubMed]

Bayat, Mojdeh, and Peter Schuntermann. 2013. Enhancing resilience in families of children with autism spectrum disorder. In Handbook of Family Resilience. Edited by D. Becvar. New York: Springer, pp. 409-24. [CrossRef]

Blake, Jasmine, Eric Rubenstein, Peng-Chou Tsai, Hafizur Rahman, Sarah Rieth, Hasmot Ali, and Li-Ching Lee. 2017. Lessons learned while developing, adapting and implementing a pilot parent-mediated behavioural intervention for children with autism spectrum disorder in rural Bangladesh. Autism 21: 611-21. [CrossRef] [PubMed]

Braun, Virginia, and Victoria Clarke. 2006. Using thematic analysis in psychology. Qualitative Research in Psychology 3: 70-101. [CrossRef]

Crane, Laura, James Chester, Lorna Goddard, Lucy Henry, and Elisabeth Hill. 2016. Experiences of autism diagnosis: A survey of over 1000 parents in the United Kingdom. Autism 20: 153-62. [CrossRef] [PubMed]

DePape, Anne-Marie, and Sally Lindsay. 2014. Parents' Experiences of Caring for a Child with Autism Spectrum Disorder. Qualitative Health Research 25: 569-83. [CrossRef] [PubMed]

Divan, Gauri, Vivek Vajaratkar, Miraj Desai, Luisa Strik-Lievers, and Vikram Patel. 2012. Challenges, coping strategies, and unmet needs of families with a child with autism spectrum disorder in Goa, India. Autism Research 5: 90-200. [CrossRef] [PubMed]

Ekas, Naomi, and Thomas Whitman. 2010. Autism symptom topography and maternal socioemotional functioning. American Journal on Intellectual and Developmental Disability 115: 234-49. [CrossRef] [PubMed]

Fido, Abdullahi, and Samira Al Saad. 2013. Psychological effects of parenting children with autism prospective study in Kuwait. Open Journal of Psychiatry 3: 5-10. [CrossRef]

Gobrial, Ereny. 2012. Mind the gap: the human rights of children with intellectual disabilities in Egypt. Journal of Intellectual Disability Research 56: 1058-64. [CrossRef] [PubMed]

Gobrial, Ereny. 2015. Attitude towards people with autism spectrum disorder in Egypt. Journal of Special Education, Zagazig University, Egypt 12: 50-73.

Gobrial, Ereny, Su McAnelly, and Patrick Shannon. 2018. The education of children and young people with autistic spectrum disorders in Egypt. British Journal of Learning Disabilities, under publication.

Hadidi, Mona, and Gamal Al Khateeb. 2015. Special Education in Arab Countries: Current challenges. International Journal of Disability, Development and Education 62: 518-30. [CrossRef]

Hock, Robert, Tina Timm, and Julie Ramisch. 2012. Parenting children with autism spectrum disorders: A crucible for couple relationships. Child E Family Social Work 17: 406-15.

Iemmi, Valentina, Karl Blanchet, Lorna Gibson, Suresh Kumar, Santosh Rath, Sally Hartley, Gudlavalleti VS Murthy, Vikram Patel, Joerg Weber, and Hannah Kuper. 2016. Community-based rehabilitation for people with physical and mental disabilities in low- and middle-income countries: A systematic review and meta-analysis. Journal of Development Effectiveness 8: 368-87. [CrossRef]

Ilias, Kartini, Jeanette Liaw, Kim Cornish, Miriam Sang-Ah Park, and Karen Golden. 2017. Wellbeing of mothers of children with "A-U-T-I-S-M" in Malaysia: An interpretative phenomenological analysis study. Journal of Intellectual \& Developmental Disability 42: 74-89.

Jegatheesan, Brinda, Peggy Miller, and Susan Fowler. 2010. Autism from a religious perspective: A study of parental beliefs in South Asian Muslim immigrant families. Focus on Autism and Other Developmental Disabilities 25: 98-109. [CrossRef]

Jenkins, Rachel, Ahmed Heshmat, Loza Nasser, Inkeri Siekkonen, and Eman Sorour. 2010. Mental Health policy and development in Egypt-Integrating mental health into health sector forms 2001-9. International Journal of Mental Health Systems 4: 17. [CrossRef] [PubMed] 
JICAPED (Japan International Cooperation Agency Planning and Evaluation Department). 2002. Country Profile on Disability Arab Republic of Egypt. Cairo: Japan International.

Karst, J. S., and A. V. Van Hecke. 2012. Parent and family impact of autism spectrum disorders: A review and proposed model for intervention evaluation. Clinical Child and Family Psychology Review 15: 247-77. [CrossRef] [PubMed]

Keenan, Mickey, Karola Dillenburger, Alvin Doherty, Tony Byrne, and Stephen Gallagher. 2010. The experiences of parents during diagnosis and forward planning for children with autism spectrum disorder. Journal of Applied Research in Intellectual Disabilities 23: 390-97. [CrossRef]

Kim, Hyun. 2012. Autism across cultures: Rethinking autism. Disability E Society 27: 535-45.

Koydemira, Selda, and Ülkü Tosuna. 2009. Impact of autistic children on the lives of mothers. Procedia Social and Behavioral Sciences 1: 2534-40. [CrossRef]

McKenzie, Judith, and Roy McConkey. 2015. Caring for adults with intellectual disability: The perspectives of family carers in South Africa. Journal of Applied Research in Intellectual Disabilities 29: 531-41. [CrossRef] [PubMed]

Mendoza, Roger. 2010. The Economics of Autism in Egypt. American Journal of Economics and Business Administration 2: 12-19. [CrossRef]

Neik, Tina T. X., Lay Wah Lee, Hui M. Low, Noel K. H. Chia, and Arnold C. K. Chua. 2014. Prevalence, diagnosis, treatment and research on autism spectrum disorders (ASD) in Singapore and Malaysia. International Journal of Special Education 29: 82-92.

Okasha, Ahmad. 2005. Mental Health in Egypt. Journal of Psychiatry and Related Sciences 42: 116-25.

Omar, Mohammad. 2014. Early intervention services as perceived by parents of children with autism in Egypt and Saudi Arabia. The International Interdisciplinary Journal of Education 3: 238-49. [CrossRef]

Omar, Tarek, Wafaa Ahmed, and Nehads Basiouny. 2017. Challenges and adjustments of mothers having children with autism. Alexandria Journal of Pediatrics 30: 120-29.

Opreaa, Crenguta, and Andreea Stan. 2012. Mothers of autistic children. How do they feel? Procedia-Social and Behavioral Sciences 46: 4191-94. [CrossRef]

Patterson, S., and V. Smith. 2011. The experience of parents of toddlers diagnosed with autism spectrum disorder in the more than words parent education program. Infants and Young Children 24: 329-42. [CrossRef]

Preece, David, and Rita Jordan. 2010. Obtaining the views of children and young people with autism spectrum disorders about their experience of daily life and social care support. British Journal of Learning Disabilities 38: 10-20. [CrossRef]

Riany, Yuliana E., Monica Cuskelly, and Pamela Meredith. 2016. Cultural Beliefs about Autism in Indonesia. International Journal of Disability, Development and Education 63: 623-40. [CrossRef]

Schieve, Laura, Stephen Blumberg, Cathrine Rice, Susanna Visser, and Coleen Boyle. 2007. The relationship between autism and parenting Stress. Paediatrics 119: S114-21. [CrossRef] [PubMed]

Smith, L., J. Greenberg, and M. Seltzer. 2012. Social Support and Well-being at Mid-Life among Mothers of Adolescents and Adults with Autism Spectrum Disorders. Journal of Autism and Developmental Disorder 42: 1818-26. [CrossRef] [PubMed]

Tilahun, Dejene, Charlotte Hanlon, Abebaw Fekadu, Bethlehem Tekola, Yonas Baheretibeb, and R. Hoekstra. 2016. Stigma, explanatory models and unmet needs of caregivers of children with developmental disorders in a low-income African country: A cross-sectional facility-based survey. BMC Health Services Research 16: 152. [CrossRef] [PubMed]

Weiss, Jonathan, Aranda Wingsiong, and Yona Lunsky. 2014. Defining crisis in families of individuals with autism spectrum disorders. Autism 18: 985-95. [CrossRef] [PubMed]

(C) 2018 by the author. Licensee MDPI, Basel, Switzerland. This article is an open access article distributed under the terms and conditions of the Creative Commons Attribution (CC BY) license (http:/ / creativecommons.org/licenses/by/4.0/). 